\title{
RINGS WITH INVOLUTION WHOSE SYMMETRIC ELEMENTS ARE REGULAR
}

\author{
CHARLES LANSKI ${ }^{1}$
}

\begin{abstract}
In this work we determine the structure of 2 torsion-free rings with involution whose nonzero symmetric elements do not annihilate one another.
\end{abstract}

In this work we obtain a rather complete structure theorem for rings with involution whose symmetric elements are not zero divisors. As a corollary we have a theorem of Osborn [2] concerning simple rings whose symmetric elements are invertible.

$R$ will always denote an associative ring with involution, denoted by *. That is, * is an anti-automorphism of $R$ of period two. Let $S=$ $\left\{r \in R \mid r^{*}=r\right\}$ be the set of symmetric elements of $R$ and $K=$ $\left\{r \in R \mid r^{*}=-r\right\}$ the set of skew-symmetric elements. We shall assume that $R$ is 2-torsion-free, and so $S \cap K=0$ and $2 R \subset S+K$ since $2 r=$ $\left(r+r^{*}\right)+\left(r-r^{*}\right)$. Let $S^{\prime}=S-\{0\}$. We shall say that $S^{\prime}$ has no zero divisors to mean: (1) $S^{\prime}$ is not empty; and (2) given $s, t \in S^{\prime}$, then $s t \neq 0$. Note that if $S^{\prime}$ were allowed to be empty then every element of $R$ has square zero, and so $R^{3}=0$. Lastly, for $a, b \in R$ let $[a, b]=a b-b a$.

The first goal is to show that if $S^{\prime}$ has no zero divisors then $R$ has a unique maximal nilpotent ideal. In fact we can assume somewhat less to begin with.

LEMMA 1. If $x \in R, 2 x=s+k$ and $x x^{*}=x^{*} x=0$, then $s^{2}=k^{2}$ and $s k=k s$.

Proof. Clearly $(2 x)(2 x)^{*}=(2 x)^{*}(2 x)=0$. Thus $(s+k)(s-k)=0=$ $(s-k)(s+k)$. Hence $s^{2}-k^{2}+k s-s k=0=s^{2}-k^{2}+s k-k s$, and so, 2(sk-ks) $=0$. Since $R$ is 2 -torsion-free, $s k-k s=0$, and thus $s^{2}=k^{2}$.

LEMMA 2. If $S^{\prime}$ has no nilpotent elements then every nil right (left) ideal $N$ of $R$ satisfies

(i) $N^{3}=0$;

(ii) $N \subset K$;

(iii) if $x \in N$ then $x^{2}=0$.

Received by the editors September 20, 1971.

AMS 1970 subject classifications. Primary 16A28; Secondary 16A48.

Key words and phrases. Ring with involution, regular symmetric elements.

${ }^{1}$ This work was supported by NSF Grant GP-29119X.

i. American Mathematical Society 1972 
Proof. If $x \in N$ then $x x^{*} \in N \cap S$. Since $S^{\prime}$ has no nilpotent elements, $x x^{*}=0$. But $\left(x^{*} x\right)^{2}=0$ so $x^{*} x=0$ as well. By Lemma 1 , if $2 x=s+k$ then $s^{2}=k^{2}$ and $s k=k s$. Suppose $x^{2}=0$. Then $4 x^{2}=0$ and $0=s^{2}+k^{2}+2 s k=$ $2\left(s^{2}+s k\right)$. Now $s k \in K$ so $s^{2}=0$. But $S^{\prime}$ has no nilpotent elements, so $s=0$. Thus $2 x$ and so $x \in K$. Next suppose that $x \in N$ and $x^{2} \in K$. Then $4 x^{2} \in K$. If $2 x=s+k$, this says $s^{2}+k^{2}+2 s k \in K$. But now $s^{2}+k^{2}=2 s^{2} \in K$. So $2 s^{2} \in K \cap S$ and as above $s=0$, so $x \in K$. We claim that $N \subset K$. If $x \in N$ then $x^{m}=0$ for some $m$. If $t \geqq m / 2$ then $\left(x^{t}\right)^{2}=0$. Since $x^{t} \in N$, by what we have shown above, $x^{t} \in K$. Let $t$ be minimal with $x^{t+p} \in K$ for $p \geqq 0$. If $t>1$ then $\left(x^{t-1}\right)^{2}=x^{t+(t-2)}$, so $\left(x^{t-1}\right)^{2} \in K$ which we have seen forces $x^{t-1} \in K$ contradicting the minimality of $t$. Thus $t=1$ and so $N \subset K$. But $x^{m}=0$ implies $\left(x^{2}\right)^{m}=0$. Since $x^{2} \in S$ we have $x^{2}=0$ for all $x \in N$. As $R$ is 2-torsion-free any subring having the square of every element zero is nilpotent of index 3 .

If $R$ is a ring with involution and $A$ is an ideal of $R$ with $A^{*}=A$ then the quotient ring $R / A$ inherits an involution defined by $(r+A)^{*}=r^{*}+A$. If $x+A$ is symmetric in $R / A$ then $x-x^{*} \in A$. Since $2 x=x+x^{*}+\left(x-x^{*}\right)$ we have $2 x+A=\left(x+x^{*}\right)+A$. Thus if $R / A$ is 2-torsion-free, twice every symmetric element comes from a symmetric element in $R$. We use this observation together with Lemma 2 to reduce to the case in which $R$ has no nil ideals.

THEOREM 3. Suppose $S^{\prime}$ has no zero divisors. Then $R$ has a unique maximal nilpotent ideal $N$ satisfying:

(i) $N \subset K$;

(ii) $N^{3}=0$;

(iii) if $x \in N$ then $x^{2}=0$;

(iv) $N$ contains all nil one-sided ideals of $R$;

(v) $R / N$ is a 2-torsion-free ring with involution containing no nil ideals;

(vi) If $S(R / N)$ are the symmetric elements of $R / N$ then $S^{\prime}(R / N)$ has no zero divisors.

Proof. Let $N$ be the sum of all nilpotent ideals of $R$. By Lemma 2, (i)-(iv) hold, and $N$ is the unique maximal nilpotent ideal of $R$. As $N^{*}=N$ we know that $R / N$ carries an induced involution and clearly contains no nil ideals. Let $T=\{r \in R \mid 2 r \in N\}$. $T$ is an ideal of $R$. If $x \in T$ then $(2 x)^{m}=$ $2^{m} x^{m}=0$. Since $R$ is 2 -torsion-free $x^{m}=0$. Hence $T$ is nil, and so, $T \subset N$. Thus $R / N$ is 2-torsion-free. Suppose $x_{1}+N, x_{2}+N \in S^{\prime}(R / N)$ and $x_{1} x_{2} \in N$. Since $2 x_{1}+N, 2 x_{2}+N \in S^{\prime}(R / N)$ and $4 x_{1} x_{2} \in N$, we may assume by the discussion above that $x_{1}, x_{2} \in S^{\prime}$. But $x_{1} x_{2} \in N$ says $\left(x_{1} x_{2}\right)^{2}=0$. Since $N \subset K,-x_{1} x_{2}=\left(x_{1} x_{2}\right)^{*}=x_{2} x_{1}$, so $0=x_{1} x_{2} x_{1} x_{2}=-x_{1}^{2} x_{2}^{2}$. Thus $x_{1}^{2}=0$ or $x_{2}^{2}=0$, and so, $x_{1}=0$ or $x_{2}=0$. 
THEOREM 4. Let $R$ be semiprime and suppose $S^{\prime}$ has no zero divisors. If $y \in R$ is a zero divisor in $R$ then $y^{*}=y^{*} y=0$. In particular every element of $S^{\prime}$ is regular in $R$.

PROOF. Let us first note that if $r^{2}=0$ then $r^{*} r r r^{*}=0$, so our assumption on $S^{\prime}$ forces, say, $r^{*} r=0$. But $\left(r r^{*}\right)^{2}=0$, and so $0=r^{*} r=r r^{*}$. Using Lemma 1 , as in Lemma 2, we conclude that $r \in K$. Suppose now that $x y=0$ but $y y^{*} \neq 0$. Since $(y R x)^{2}=0$ we have $y R x \subset K$. Thus if $r \in R,-y r x=x^{*} r^{*} y^{*}$, which implies $x^{*} r^{*} y^{*} y=0$. Also $\left(y^{2} R x\right)^{2}=0$ and so we obtain $x^{*} r^{*}\left(y^{*}\right)^{2} y^{2}=$ 0 . Thus $R y^{*} y x^{*}$ and $R\left(y^{*}\right)^{2} y^{2} x^{*}$ are nil left ideals of $R$ of index 2. By Levitzki's Theorem [1, Lemma 1.1] these ideals are zero, since $R$ is semiprime. Thus $y^{*} y x^{*}=\left(y^{*}\right)^{2} y^{2} x^{*}=0$. Now $x y=0$ says $(y x)^{2}=0$, so $y x \in K$. Therefore $-y^{x} x=x^{*} y^{*}$. Using $y^{*} y x^{*}=0$ we have $y^{*} y x^{*} y^{*}=0$, and so $-y^{*} y^{2} x=0$. Together with $\left(y^{*}\right)^{2} y^{2} x^{*}=0$ we obtain $\left(y^{*}\right)^{2} y^{2}\left(x+x^{*}\right)=0$. Since $\left(y^{*}\right)^{2} y^{2} \in S$ either $x+x^{*}=0$ or $\left(y^{*}\right)^{2} y^{2}=0$. In the latter case $\left(y y^{*}\right)\left(y^{*} y\right)\left(y y^{*}\right)\left(y^{*} y\right)=0$. Since $\left(y y^{*} v^{*} y\right)^{2}=0$ it is in $K$, so we obtain $\left(y y^{*}\right)^{2}\left(y^{*} y\right)^{2}=0$. Hence $y y^{*}=0$ or $y^{*} y=0$, both of which are impossible. Thus we must have $x+x^{*}=0$, so $x \in K$. Since $x^{2} y_{y^{*}}=0$ we have $x^{2}=0$.

From above we have $x^{*} R y^{*} y=0$, and so $x R y^{*} y=0$. But $\left(y^{*} y\right)\left(y^{*} y\right)^{*} \neq 0$ so $x R \subset K$. Hence $-x r=-r^{*} x$ for all $r \in R$. Thus $x r x t=r^{*} x^{2} t=0$. Since $R$ is semiprime and $(x R)^{2}=0$ we must have $x=0$. Thus if $x y=0$ for $x, y \neq 0$ we must have $y y^{*}=0$, and so $y^{*} y=0$. If $y x=0$ to begin with then $x^{*} y^{*}=0$, so again $y^{*} y=y y^{*}=0$.

LEMMA 5. If $R$ is semiprime and $S^{\prime}$ has no zero divisors then either $S \subset Z$, the center of $R$, or $R$ has no nilpotent elements.

Proof. As in Theorem 4 if $x \in R$ and $x^{2}=0$ then $x \in K$. Further $(x R x)^{2}=0$, so $x R x \subset K$. As $x \in K$ we have $x S x \subset K \cap S=0$. If $s \in S$ then $(x s)^{2}=0$ says $x s \in K$, so $-x s=-s x$ and $x$ commutes elementwise with $S$. Suppose $S \notin Z$. Then $2 S \notin Z$. Let $(2 S)^{-}$be the subring of $2 R$ generated by $2 S$. Since $2 R \subset S+K$, by an easy argument $\left[1\right.$, p. 10] $(2 S)^{-}$is a Lie ideal of $2 R$. Since $2 R$ is a 2 -torsion-free semiprime ring and $2 S$ is not in its center we have that $(2 S)^{-}$must contain a nonzero ideal of $2 R$ [1. Lemma 1.3]. Let $I$ be the sum of all such ideals. Clearly $I^{*}=I$, and also, since $x$ commutes with $S, x$ commutes elementwise with $I$. Thus $(2 x) I 2 x=0$ and $2 x I$ is a nilpotent ideal in $2 R$, which is impossible. Hence $x I=0$. If $I \cap S \neq 0$ then $x=0$ since Theorem 4 implies that elements of $S^{\prime}$ are regular in $R$. Assume then that $I \cap S=0$. Since $I^{*}=I$, for $y \in I, y+y^{*} \in I \cap S=0$. Thus $I \subset K$. But now if $y \in I, y^{2} \in I \cap S=0$. Since $I$ is nil of index 2. its cube is zero, contradicting $2 R$ a semiprime ring. The net result is that $R$ can contain no elements of square zero, and so, no nilpotent elements. 
THEOREM 6. Let $R$ be semiprime and suppose $S^{\prime}$ has no zero divisors. Then one of the following hoids:

(i) $R$ is a domain;

(ii) $S^{\prime} \subset Z$, the center of $R$, and $R\left(S^{\prime}\right)^{-1}$, the localization of $R$ at $S^{\prime}$, is the complete $2 \times 2$ matrix ring over a field;

(iii) $R$ is a subring of $A \oplus A^{\mathrm{op}}$, where $A \cong R / P$ is a domain, $A^{\mathrm{op}}$ is its opposite ring, $P \cup P^{*}$ is the set of zero divisors of $R$, and * in $R$ is induced by interchanging co-ordinates in $A \oplus A^{\text {op }}$.

Proof. By Lemma 5 either $S^{\prime} \subset Z$ or $R$ has no nilpotent elements. If $R$ is commutative, then since $R$ is semiprime, it has no nilpotent elements. Thus we may consider two cases: one where $S^{\prime} \subset Z$ and $R$ is not commutative, and the other where $R$ has no nonzero nilpotent elements.

To start with, suppose $S^{\prime} \subset Z$ and $R$ is not commutative. Since $S \subset Z$ we have $[R, R] \subset K$. Suppose $I \neq 0$ is an ideal of $R$ consisting only of zero divisors. Note that by Theorem 4 there is no distinction between left, right, and two-sided zero divisors. If $I \notin Z$ then $[I, R] \neq 0$. Now $[I, R] \subset I \cap K$, so it consists of zero divisors in $K$. If $x \in[I, R]$ then $x^{2} \in S$, so $x^{2}=0$, using Theorem 4 again. But for any $r \in R, x r-r x \in[I, R]$, so $0=(x r-r x)^{2} x$. Thus $x R$ is a nil right ideal of $R$ of index 3 . Since $R$ is semiprime, $x=0$. Hence if $I$ consists of zero divisors, $I \subset Z$. Now suppose that $R$ is not prime. Let $P$ be an ideal of $R$ maximal with respect to exclusion of the multiplicative semigroup of regular elements of $R$. By the usual argument, $P$ is prime and so $P \neq 0$ since $R$ is not prime. Since $P$ consists of zero divisors $P \subset Z$ by what was just shown. Hence $[P, R]=0$. But this implies $P[R, R]=0$. Since $P \neq 0,[R, R]$ must consist of zero divisors. As above, we have for $x \in[R, R]$, that $x^{2} \in S$ and is a zero divisor, so $x^{2}=0$. If $x \in[R, R]$ and $x \neq 0$ then $(x r-r x)^{2} x=0$ and again we obtain a contradiction. Thus $[R, R]=0$ and $R$ is commutative. Since we are assuming that $R$ is not commutative, we must assume that $R$ is prime.

By Theorem 4 every element of $S^{\prime}$ is regular in $R$. As $S^{\prime} \subset Z$, it is a multiplicatively closed set, and so, we can consider the localization $R\left(S^{\prime}\right)^{-1}$, which is still a 2 -torsion-free prime ring. Furthermore we can extend ${ }^{*}$ to $R\left(S^{\prime}\right)^{-1}$ by defining $\left(r s^{-1}\right)^{*}=r^{*} s^{-1}$. A straightforward verification yields that this is indeed an involution extending $*$, with the property that $r s^{-1}$ is symmetric exactly when $r$ is.

If $x$ is a non-zero-divisor in $R\left(S^{\prime}\right)^{-1}$ then $x x^{*}$ is a unit, so $x$ itself is a unit. Thus any proper ideal $I$ of $R\left(S^{\prime}\right)^{-1}$ must consist of zero divisors. But as we have seen above this forces $I \subset Z$. Since $R\left(S^{\prime}\right)^{-1}$ is prime, either $I=0$ or $R\left(S^{\prime}\right)^{-1}$ is commutative. As we are assuming that $R$ is not commutative we have that $R\left(S^{\prime}\right)^{-1}$ is simple. Now $S^{\prime} \subset Z$ implies that if $x \in R\left(S^{\prime}\right)^{-1}$ then $x^{2}-\left(x+x^{*}\right) x+x^{*} x=0$, so $R\left(S^{\prime}\right)^{-1}$ is quadratic over its center. Hence 
$R\left(S^{\prime}\right)^{-1}$ satisfies a polynomial identity of degree five over its center. Since $R\left(S^{\prime}\right)^{-1}$ is simple it must be either a division ring or the complete $2 \times 2$ matrix ring over a field by Kaplansky's Theorem. In the first case $R$ is a domain and in the second we have (ii).

We now consider the second main case where $R$ has no nonzero nilpotent elements. If $R$ is prime, clearly it is a domain, so we obtain (i). We may henceforth assume that $R$ is not prime. Let $P$ be an ideal of $R$ maximal with respect to exclusion of the multiplicative semigroup of regular elements of $R$. As above, $P$ is prime and $P \neq 0$ since $R$ is not prime. Further, $P$ consists of zero divisors, so $P \cap S=0$. Suppose $x y=0$. Then $(y R x)^{2}=0$ and we have $y R x=0$. Since $P$ is prime either $x \in P$ or $y \in P$. Suppose $x \notin P$. Since $x x^{*}=0$, by Theorem 4, we have $x^{*} \in P$. Thus if $x$ is any zero divisor either $x \in P$ or $x^{*} \in P$. This says that $P \cup P^{*}$ is the set of zero divisors of $R$.

Suppose $x^{2} \in P$ but $x \notin P$. Now $x^{2} \in P$ means that $x$ is a zero divisor and so $x x^{*}=x^{*} x=0$ by Theorem 4 . Since $x \notin P$ we have $x^{*} \in P$. If $2 x=s+k$ then $2 x^{*}=s-k$. By Lemma $1, s^{2}=k^{2}$ and $s k=k s$. Thus $\left(2 x^{*}\right)^{2}=s^{2}+k^{2}-$ $2 s k \in P$ and $4 x^{2}=s^{2}+k^{2}+2 s k \in P$. We conclude that $2\left(s^{2}+k^{2}\right)=4 s^{2} \in$ $P \cap S=0$, and so, $s=0$. But $k^{2}=s^{2}=0$ and $R$ has no nilpotent elements, so $x=0$. We have shown that $R / P$ is a prime ring with no elements of square zero, so it is a domain.

If $x \in P \cap P^{*}$ then $x \in P^{*}$ implies $x^{*} \in P$. Hence $x+x^{*}$ and $x x^{*}$ are in $P \cap S=0$. Thus $0=x x^{*}=-x^{2}$, so $x=0$. Hence $R$ is a subdirect sum of $R / P$ and $R / P^{*}$ via $r \mapsto\left(r+P, r+P^{*}\right)$. Now $R / P^{*}$ is naturally isomorphic to $(R / P)^{\circ \mathrm{p}}$ via the map $r+P^{*} \mapsto r^{*}+P$, which is clearly a bijection and additive. Further $r t+P^{*} \mapsto(r t)^{*}+P=t^{*} r^{*}+P$ and $\left(r^{*}+P\right) \circ\left(t^{*}+P\right)=$ $r^{*} \circ t^{*}+P=t^{*} r^{*}+P$. If $A=R / P$ we have that $R$ is a subdirect sum of $A$ and $A^{\text {p }}$ via $r \mapsto\left(r+P, r^{*}+P\right)$. Cleaily ${ }^{*}$ in $R$ is induced by the involution in $A \oplus A^{\mathrm{op}}$ which interchanges co-ordinates.

COROLLARY 7. If $R$ is semiprime and $S^{-}$, the subring generated by $S$, is a domain, then $R$ is either commutative, a domain, or $S \subset Z$ and $R\left(S^{\prime}\right)^{-1}$ is a complete $2 \times 2$ matrix ring over a field.

Proof. As in Theorem 6, if $S \subset Z$ then $R$ is either commutative or $R\left(S^{\prime}\right)^{-1}$ is a complete $2 \times 2$ matrix ring over a field. Hence we may assume that $S \notin Z$. and so, that $R$ has no nilpotent elements. If $R$ is prime it must then be a domain. Suppose $R$ is not prime and let $P$ be an ideal maximal with respect to exclusion of regular elements. Then $P \neq 0$ and consists of zero divisors. Since $S \notin Z$ by Lemma 1.3 of [1] we have, as in Lemma 5, that $(2 S)^{-}$contains a nonzero ideal of $2 R$. If $I$ is the sum of all such ideals, then $I \subset(2 S)^{-}, I^{*}=I$. and $I$ consists only of regular elements of $R$. For if $x \in I-\{0\}$, then $x^{*} \in I$ so $x x^{*}$ and $x^{*} x$ are nonzero, $(2 S)^{-}$being a domain, and so regular in $R$ by Theorem 4 . But $P I \subset P \cap I=0$ since $P$ consists of 
zero divisors. Thus $P=0$. This contradiction shows that $R$ must be prime, and so, a domain.

Next we consider some examples to show that the various possibilities in Theorem 6 can occur.

Let $R=D[x, y]$ the polynomial ring in two indeterminates over any domain $D$ of char $\neq 2$. Define ${ }^{*}$ by interchanging $x$ and $y$. For a noncommutative example one can take the quaternions over the integers with the usual conjugation as the involution. By considering the free algebra in at least two indeterminates over a field of char $\neq 2$, and defining * to reverse the order of the indeterminates in each monomial, we have a domain whose symmetric elements are not central.

An example for the second case of the theorem would be to let $R=D_{2}$, where $D$ is any commutative domain of char $\neq 2$ and define * by $\left(\begin{array}{ll}a & b \\ c & d\end{array}\right) *=\left(\begin{array}{cc}d & -b \\ -c & a\end{array}\right)$.

For the third case let $R_{1}=F[x, y, z]$ and let $J$ be the ideal generated by $x y$. Set $R=R_{1} / J$ and define ${ }^{*}$ to interchange $x$ and $y$, and fix $z$. A typical symmetric element of $R$ is $f(z)+\sum_{i=0}^{n}\left(p_{i}(x)+p_{i}(y)\right) z^{i}$ where $p_{i}$ has no constant term. The ideal $P$ in Theorem 6 can be taken to be $(x)$, so $P^{*}=(y)$. Since $P+P^{*} \neq R, R$ is indeed a subdirect sum as indicated and not a direct sum.

To get a noncommutative example for the third case let $F=$ $(F[x, y] /(x y))\{w, t\}$ and define ${ }^{*}$ to interchange $x$ and $y$ and reverse the order of each monomial in $w$ and $t$. Again if $P=(x)$ and $P^{*}=(y)$ then $R$ is a subdirect sum of $R /(x)$ and $R /(y)$ but $(x)+(y) \neq R$ implies that $R$ is not a direct sum.

For a nonsemiprime example in any of the above cases, let $R$ be one of the above examples and consider $R\{x, y, z\}$, the polynomial ring over $R$ in 3 noncommuting indeterminates. Consider $R$ to be a homomorphic image of the free algebra with identity over $F$ in the indeterminates $\left\{t_{i}\right\}$. Let $I$ be the ideal in $R\{x, y, z\}$ generated by $t_{i} x, t_{i} y$, and $t_{i} z$ for all $i$ and by all squares in $F\{x, y, z\}$ (without constants). Consider $A=R\{x, y, z\} / I$. Extend * to $A$ by interchanging the order of indeterminates of monomials in $x, y$, and $z$ and also sending $x, y, z$ to their negatives. If $N$ is the ideal generated by $x, y$, and $z$, then $N \subset K, N^{3}=0$ as in Theorem 3 , and $A / N \cong R$.

Finally; we consider the situation in which $R$ has an identity 1 and every element of $S^{\prime}$ is invertible. Again we will assume that $S^{\prime}$ is not empty.

THEOREM 8. Suppose $R$ is semiprime with 1 and that every element of $S^{\prime}$ is $a$ unit in $R$. Then one of the following holds:

(i) $S \subset Z$ and $R$ is the complete $2 \times 2$ matrix ring over a field;

(ii) $R$ is a division ring;

(iii) $R \equiv D \oplus D^{\mathrm{op}}$ where $D$ is a division ring, $D^{\mathrm{op}}$ its opposite ring, and * in $R$ is induced by interchanging co-ordinates in $D \oplus D^{\mathrm{op}}$. 
Proof. Let us examine the possibilities which can occur by Theorem 6 . First suppose $R$ is a domain. If $x \in R$ and $x \neq 0$ then $x^{*} x$ and $x x^{*} \in S^{\prime}$, so $x$ is a unit, and $R$ is a division ring. Next assume (ii) of Theorem 6 holds. Since every element of $S^{\prime}$ is invertible, $R\left(S^{\prime}\right)^{-1} \cong R$, so $R$ is the complete $2 \times 2$ matrix ring over a field. In the last case of Theorem 6 , we need only show that $R \cong A \odot A^{\text {op }}$ and that $A$ is a division ring. Note first that $\left(P+P^{*}\right) \cap$ $S \neq 0$. Otherwise $P \subset K$ and since $P$ consists of zero divisors in $R, x \in P$ implies $x^{2} \in P \cap S=0$. But if $P$ is nil of index 2 it is a nilpotent ideal of $R$, a semiprime ring. Thus we must have $\left(P+P^{*}\right) \cap S \neq 0$. Since $S^{\prime}$ consists of units, $P+P^{*}=R$ and so $R \cong A \subseteq A^{\text {op }}$ by the Chinese Remainder Theorem. To show that $R / P$ is a division ring it suffices to show that if $x \in R, x \neq 0$ is a zero divisor in $R$ then $x \in P$ or $x+P$ is a unit in $R / P$. Suppose then, that $x \notin P$ and $x$ is a zero divisor in $R$. By Theorem $4, x x^{*}=x^{*} x=0$ and so if $x=s+k$ we have by Lemma 1 that $s^{2}=k^{2}$ and $s k=k s$ (note that we now have $\frac{1}{2} \in R$ ). Since $R$ is a subring of the direct sum of two domains, $R$ has no nilpotent elements. Hence if $s=0$, then $k^{2}=s^{2}=0$, so $x=0$. Therefore $s \neq 0$ and we may consider $x s^{-1} / 2=\frac{1}{2}\left(1+s^{-1} k\right)=e$. Now $e^{2}=e \neq 0$ and $e^{*}=1-e$. Since $e$ or $e^{*}$ is in $P$ we must have $e^{*} \in P$ if $x \notin P$. But this says that $(x+P)\left(s^{-1} / 2+P\right)=1+P$, so $x+P$ is a unit in $R / P$. Thus $R / P$ is a division ring and the proof is complete.

COROLLARY 9 (OSBORN). If $R$ is a simple ring with 1 and every element of $S^{\prime}$ is invertible, then $R$ is either a division ring or $\operatorname{dim}_{z} R \leqq 4$.

\section{REFERENCES}

1. I. N. Herstein, Topics in ring theory, Univ. of Chicago Press, Chicago, Ill., 1969.

2. N. Jacobson, Structure theory for a class of Jordan algebras, Proc. Nat. Acad. Sci. U.S.A. 55 (1966), 243-251. MR 33 \#1337.

Department of Mathematics, University of Southern California, Los Angeles, CALIFornia 90007 